\title{
Training Data Cleaning for Text Classification
}

\author{
Andrea Esuli and Fabrizio Sebastiani \\ Istituto di Scienza e Tecnologia dell'Informazione \\ Consiglio Nazionale delle Ricerche \\ Via Giuseppe Moruzzi 1 - 56124 Pisa, Italy \\ \{andrea.esuli,fabrizio.sebastiani\}@isti.cnr.it
}

\begin{abstract}
In text classification (TC) and other tasks involving supervised learning, labelled data may be scarce or expensive to obtain; strategies are thus needed for maximizing the effectiveness of the resulting classifiers while minimizing the required amount of training effort. Training data cleaning (TDC) consists in devising ranking functions that sort the original training examples in terms of how likely it is that the human annotator has misclassified them, thereby providing a convenient means for the human annotator to revise the training set so as to improve its quality. Working in the context of boosting-based learning methods we present three different techniques for performing TDC and, on two widely used TC benchmarks, evaluate them by their capability of spotting misclassified texts purposefully inserted in the training set.
\end{abstract}

\section{Introduction}

In many applicative contexts involving supervised learning, labelled data may be scarce or expensive to obtain. In such situations, once we have trained the classifiers with the available training data (and tested them on the test data, and/or applied them to the unlabelled data that need to be classified), we are often left with the issue of how to improve the effectiveness of the existing classifiers, given that the amount of humanpower needed to perform further labelling is limited. One potential solution is to apply (computer-assisted) training data cleaning (TDC). TDC techniques attempt to minimize the additional effort required from human annotators. Indeed, training data often contain misclassified items, sometimes as a result of lack of experience on the part of the junior annotators who have performed the labelling, sometimes as a result of tight time constraints under which the labelling activity has been performed. A good TDC technique top-ranks the training examples with the highest likelihood of being misclassified, which allows the human annotator to improve the quality of the training set by double-checking the labels attached to the training examples, starting with the ones most likely to be erroneous, and working down the ranked list until s/he sees fit. We present three different techniques for performing TDC in TC, and test them using a boosting-based supervised learning device that generates confidence-rated predictions. The reason we are using this device is that it has two features that allow us to exemplify our TDC techniques particularly 
well, i.e., (i) it allows for a notion of confidence in the classifier's classification decisions; and (ii) the classifier it generates is actually a classifier committee.

\section{Preliminaries}

This work attempts to identify good TDC techniques for text classification (aka text categorization - TC), and for multi-label text classification (MLTC) in particular. Given a set of textual documents $D$ and a predefined set of classes (aka labels, or categories) $C=\left\{c_{1}, \ldots, c_{m}\right\}$, MLTC can be defined as the task of estimating an unknown target function $\Phi: D \times C \rightarrow\{-1,+1\}$, that describes how documents ought to be classified, by means of a function $\hat{\Phi}: D \times C \rightarrow\{-1,+1\}$ called the classifier 1 ; here, +1 and -1 represent membership and non-membership of the document in the class. As usual, we accomplish MLTC by generating $m$ independent binary classifiers $\hat{\Phi}^{j}: D \rightarrow\{-1,+1\}$, one for each $c_{j} \in C$, entrusted with the task of deciding whether a document belongs or not to class $c_{j}$.

As the learning device we use a boosting-based learner, called MP-Boost [1]; MP-Boost is a variant of AdABoost.MH [2] optimized for multi-label settings, which has been shown in [1] to obtain considerable effectiveness improvements with respect to ADABoost.MH.

MP-Boost works by iteratively generating, for each class $c_{j}$, a sequence $\hat{\Phi}_{1}^{j}, \ldots, \hat{\Phi}_{S}^{j}$ of classifiers (called weak hypotheses). A weak hypothesis is a function $\hat{\Phi}_{s}^{j}: D \rightarrow \mathbf{R}$, where $D$ is the set of documents and $\mathbf{R}$ is the set of real numbers. The sign of $\hat{\Phi}_{s}^{j}\left(d_{i}\right)$ (denoted by $\operatorname{sgn}\left(\hat{\Phi}_{s}^{j}\left(d_{i}\right)\right)$ ) represents the binary decision of $\hat{\Phi}_{s}^{j}$ on whether $d_{i}$ belongs to $c_{j}$, i.e. $\operatorname{sgn}\left(\hat{\Phi}_{s}^{j}\left(d_{i}\right)\right)=+1$ (resp., -1$)$ means that $d_{i}$ is believed to belong (resp., not to belong) to $c_{j}$. The absolute value of $\hat{\Phi}_{s}^{j}\left(d_{i}\right)$ (denoted by $\left|\hat{\Phi}_{s}^{j}\left(d_{i}\right)\right|$ ) represents instead the confidence that $\hat{\Phi}_{s}^{j}$ has in this decision, with higher values indicating higher confidence.

At each iteration $s$ MP-Boost tests the effectiveness of the most recently generated weak hypothesis $\hat{\Phi}_{s}^{j}$ on the training set, and uses the results to update a distribution $D_{s}^{j}$ of weights on the training examples. The initial distribution $D_{1}^{j}$ is uniform. At each iteration $s$ all the weights $D_{s}^{j}\left(d_{i}\right)$ are updated, yielding $D_{s+1}^{j}\left(d_{i}\right)$, so that the weight assigned to an example correctly (resp., incorrectly) classified by $\hat{\Phi}_{s}^{j}$ is decreased (resp., increased). The weight $D_{s+1}^{j}\left(d_{i}\right)$ is thus meant to capture how ineffective $\hat{\Phi}_{1}^{j}, \ldots, \hat{\Phi}_{s}^{j}$ have been in guessing the correct $c_{j}$-assignment of $d_{i}$ (denoted by $\Phi^{j}\left(d_{i}\right)$ ), i.e., in guessing whether training document $d_{i}$ belongs to class $c_{j}$ or not. By using this distribution, MP-BoosT generates a new weak hypothesis $\hat{\Phi}_{s+1}^{j}$ that concentrates on the examples with the highest weights, i.e. those that had proven harder to classify for the previous weak hypotheses. The overall prediction on whether $d_{i}$ belongs to $c_{j}$ is obtained as a sum $\hat{\Phi}^{j}\left(d_{i}\right)=\sum_{s=1}^{S} \hat{\Phi}_{s}^{j}\left(d_{i}\right)$ of the predictions of the weak hypotheses. The final classifier $\hat{\Phi}^{j}$ is thus a committee of $S$ classifiers, each classifier casting a

${ }^{1}$ Consistently with most mathematical literature we use the caret symbol $\left({ }^{\wedge}\right)$ to indicate estimation. 
weighted vote (the vote being the binary decision $\operatorname{sgn}\left(\hat{\Phi}_{s}^{j}\left(d_{i}\right)\right)$, the weight being the confidence $\left.\left|\hat{\Phi}_{s}^{j}\left(d_{i}\right)\right|\right)$ on whether $d_{i}$ belongs to $c_{j}$. For the final classifier $\hat{\Phi}^{j}$ too, $\operatorname{sgn}\left(\hat{\Phi}^{j}\left(d_{i}\right)\right)$ represents the binary decision as to whether $d_{i}$ belongs to $c_{j}$, while $\left|\hat{\Phi}^{j}\left(d_{i}\right)\right|$ represents the confidence in this decision.

\section{Three Techniques for Training Data Cleaning}

In the following, by a TDC technique we will mean a technique that, given a training set $T r$ and a class $c_{j}$, produces a ranking $r_{j}(T r)$ in which the elements of $T r$ are sorted in decreasing order of their likelihood of being mislabelled for $c_{j}$. Different techniques correspond to different ways of estimating this likelihood.

We now present three alternative TDC techniques. For each $c_{j} \in C$, the first technique (that we dub the confidence-based technique - CON, in short) consists in (i) training the classifier $\hat{\Phi}^{j}$ on $T r$; (ii) reclassifying $T r$ by means of $\hat{\Phi}^{j}$; and (iii) ranking $T r$ in increasing order of $\hat{\Phi}^{j}\left(d_{i}\right) \cdot \Phi^{j}\left(d_{i}\right)$ value. Note that, while $\Phi^{j}\left(d_{i}\right)$ is a value in $\{-1,+1\}, \hat{\Phi}^{j}\left(d_{i}\right)$ is a value in $(-\infty,+\infty)$, so $\hat{\Phi}^{j}\left(d_{i}\right) \cdot \Phi^{j}\left(d_{i}\right)$ is also in $(-\infty,+\infty)$. A positive (resp., negative) value of $\hat{\Phi}^{j}\left(d_{i}\right) \cdot \Phi^{j}\left(d_{i}\right)$ indicates correct (resp., incorrect) classification, while a high (resp., low) absolute value of $\hat{\Phi}^{j}\left(d_{i}\right) \cdot \Phi^{j}\left(d_{i}\right)$ indicates that this classification decision has been taken with high (resp., low) confidence. CON thus corresponds to (a) top-ranking the examples $d_{i} \in \operatorname{Tr}$ that $\hat{\Phi}^{j}$ has misclassified, in decreasing order of the confidence $\left|\hat{\Phi}^{j}\left(d_{i}\right)\right|$ with which $\hat{\Phi}^{j}$ has taken its decision, and (b) appending to this list the examples $d_{i} \in \operatorname{Tr}$ that $\hat{\Phi}^{j}$ has correctly classified, in increasing order of the confidence $\left|\hat{\Phi}^{j}\left(d_{i}\right)\right|$. The rationale of this technique is that, if $\hat{\Phi}^{j}$ has misclassified a training example $d_{i}$ with high confidence, this means that the $c_{j}$-assignment made to $d_{i}$ by the human annotator is highly at odds with the $c_{j}$-assignments that the human annotator has made for the other training examples. This indicates that the human annotator may well have misclassified $d_{i}$ for $c_{j}$.

For each $c_{j} \in C$, the second technique (that we dub the nearest neighbours technique - NN) consists in ranking the training examples in terms of how inconsistent their $c_{j}$-assignment is with the $c_{j}$-assignments of their $k$ nearest neighbours, for a predefined $k$. More formally, this consists in (i) computing, for each $d_{i} \in T r$, the value

$$
\zeta\left(d_{i}, c_{j}\right)=\sum_{d_{z} \in \operatorname{Tr}_{k}\left(d_{i}\right)} \operatorname{sim}\left(d_{i}, d_{z}\right) \cdot \Phi^{j}\left(d_{z}\right)
$$

where $\operatorname{sim}(\cdot, \cdot)$ denotes a measure of similarity between documents and $\operatorname{Tr}_{k}\left(d_{i}\right)$ denotes the $k$ training examples most similar to $d_{i}$; and (ii) ranking $\operatorname{Tr}$ in increasing order of $\zeta\left(d_{i}, c_{j}\right) \cdot \Phi^{j}\left(d_{i}\right)$ value. For class $c_{j}$, the examples $d_{i}$ with $c_{j}$-assignments highly consistent with the $c_{j}$-assignments of their neighbours will have high $\zeta\left(d_{i}, c_{j}\right) \cdot \Phi^{j}\left(d_{i}\right)$ values, which means that the top-ranked examples (which are the ones with the lowest $\zeta\left(d_{i}, c_{j}\right) \cdot \Phi^{j}\left(d_{i}\right)$ values) will be the ones with $c_{j}$-assignments most dissimilar from those of their closest neighbours. 
Equation (1), of course, is that of the standard distance-weighted $k$-NN learning device, the only difference being that, while in the standard case $\Phi^{j}\left(d_{z}\right)$ ranges on $\{0,1\}$, in our case it ranges on $\{-1,+1\}$, which means that neighbours with a negative $c_{j}$-assignment weigh negatively on $\zeta\left(d_{i}, c_{j}\right)$.

For each $c_{j} \in C$, the third technique (that we dub the committee-based technique - COM) consists in (i) training the classifier $\hat{\Phi}^{j}$ on $T r$; (ii) reclassifying $T r$ by means of $\hat{\Phi}^{j}$; and (iii) ranking $T r$ in increasing order of $\Delta\left(\hat{\Phi}^{j}\left(d_{i}\right)\right) \cdot \operatorname{sgn}\left(\hat{\Phi}^{j}\left(d_{i}\right)\right)$. $\Phi^{j}\left(d_{i}\right)$ value, where $\Delta\left(\hat{\Phi}^{j}\left(d_{i}\right)\right)$ is a measure of the disagreement among the $S$ members of $\hat{\Phi}^{j}$ on whether $d_{i}$ belongs to $c_{j}$ or not. This technique is based on the intuition that the examples most in need of double-checking are the ones which $\hat{\Phi}^{j}$ has misclassified (i.e., are such that $\operatorname{sgn}\left(\hat{\Phi}^{j}\left(d_{i}\right)\right) \cdot \Phi^{j}\left(d_{i}\right)=-1$ ) with the most widespread agreement among its $S$ members. In other words, if the information that a training example provides to the training process is so inconsistent with that provided by the other training data, as to have the members of the generated classifier committee misclassify the example, and with widespread agreement, then it is likely that the example might be mislabelled. This technique will thus top-rank the training examples that the committee has misclassified and on which the $S$ members of the committee agree most. The key difference between the first technique $(\mathrm{CON})$ and this technique is that here the confidence that a classifier committee has in a certain decision is taken to coincide with the level of (weighted) agreement among its members, and not with the (weighted) sum of the individual opinions. As a measure of disagreement among the $S$ members of the committee we have chosen to use standard deviation (denoted $\sigma$ ). This is a natural choice, given that the values $\hat{\Phi}_{1}^{j}\left(d_{i}\right), \ldots, \hat{\Phi}_{S}^{j}\left(d_{i}\right)$ are real numbers: standard deviation thus allows to measure disagreement by taking into account not only the polarity $\operatorname{sgn}\left(\hat{\Phi}_{s}^{j}\left(d_{i}\right)\right)$ of each member's decision, but also its confidence level $\left|\hat{\Phi}_{s}^{j}\left(d_{i}\right)\right|$, so that two members with views of different polarity are taken to disagree more if they are highly confident in their views, and less if they are not.

Actually, there is a fourth technique (that we dub the distribution-based technique - DIS) that might come to mind. For each $c_{j} \in C$, this technique consists in (i) training the classifiers $\hat{\Phi}^{j}$ on $T r$, and (ii) ranking the examples $d_{i} \in T r$ in decreasing order of the $D_{S}^{j}\left(d_{i}\right)$ value that MP-Boost has produced as a side effect of the learning process. The rationale of this technique is that, since the training examples that maximize $D_{S}^{j}\left(d_{i}\right)$ are the ones that have turned out the most difficult to make sense of during the boosting iterations, they are thus the ones whose $c_{j}$-assignment is most highly at odds with the $c_{j}$-assignment of the other training examples. The problem with the DIS technique is that it turns out to be equivalent to our first technique $(\mathrm{CON})$, in the sense that $\mathrm{CON}$ and DIS always generate identical rankings, a fact that had never been noted in the literature 2 . The only advantage that DIS provides over $\mathrm{CON}$ is thus that there is

\footnotetext{
${ }^{2}$ We discovered this fact experimentally in the course of this work. A conversation with Robert Schapire, one of the "fathers" of boosting, later revealed that, while this phenomenon had never been observed before, an a posteriori justification can be found for it in the theory that underlies the ADABoost.MH algorithm, of which MP-Boost is a variant.
} 
no need to reclassify the training examples by means of $\hat{\Phi}^{j}$, since the information needed for ranking is already available after training has occurred.

Before discussing the experiments it is worthwhile noting that, although we have described these techniques in the context provided by a boosting-based learner which generates confidence-rated predictions, all of these techniques can be used also in connection with other learning devices. More specifically, CON only needs the classifier to return a score of confidence in its own decision, NN has no specific requirements, and COM requires the classifier to consist of a committee of classifiers. Moreover, the discussed equivalence between CON and DIS has the practical consequence of making available a technique equivalent to DIS to learning devices not based on boosting.

\section{Experiments}

In order to test our TDC techniques we use a standard MLTC dataset $\Omega=$ $\langle T r, T e\rangle$ split into a training set $T r$ and a test set $T e$. We assume that $\operatorname{Tr}$ contains no misclassified examples, and we simulate the presence of misclassified training examples by artificially "perturbing" a small number $m$ of training examples; we call the value $p=\frac{m}{|T r|}$ the perturbation ratio. In what follows, "perturbing a training example $d_{i}$ for class $c_{j}$ " means changing its $c_{j}$-assignment, from positive to negative (in this case we call $d_{i}$ a false negative for $c_{j}$ ) or from negative to positive (a false positive); by $\widehat{T r}$ we denote the training set after perturbation.

We test two different perturbation techniques, which we call random perturbation ( $\mathrm{RP}$ ) and targeted perturbation ( $\mathrm{TP})$. As the name implies, in RP the training examples to perturb are picked at random from $\mathrm{Tr}$. The same training examples ( $x \%$ of the entire lot) are perturbed for all classes $c_{j} \in C$. TP is instead obtained by (i) training the classifiers $\hat{\Phi}^{j}$ on $\operatorname{Tr}$, (ii) reclassifying $T r$ by means of them, (iii) ranking, for each $c_{j} \in C$, the reclassified examples in increasing order of the confidence $\left|\hat{\Phi}^{j}\left(d_{i}\right)\right|$ that $\hat{\Phi}^{j}$ had in classifying them, and (iv) perturbing the top-ranked ones, in number equal to $x \%$ of the training examples. The rationale of this technique is that the training examples that $\hat{\Phi}^{j}$ classifies with low confidence are more likely to be "borderline" examples for $c_{j}$. As a result, these examples are the ones that, should they be manually labelled, would have the highest probability of being misclassified (either due to lack of experience or to lack of adequate time) by a human annotator. In other words, while RP simulates the perturbation of a training set that might derive from, say, file corruption, TP simulates the perturbation that might derive from lack of experience, or lack of care, on the part of the human annotator who has labelled the training set. Unlike in RP, in TP we allow different training examples to be perturbed for different classes $c_{j} \in C$, since the same document might be controversial, or "borderline", for one class but not for others.

In order to determine which among the three TDC techniques of Section 3 is the best we will measure how good each technique is at ranking $\widehat{T r}$ in such a way that the perturbed training examples are placed at the top of the ranking. To this end, it seems natural to adopt one of the measures routinely used for evaluating 
ad-hoc (ranked) retrieval. Of course, ad-hoc retrieval is all about ranking the "good" (i.e., relevant to the information need) examples higher than the bad ones, while TDC aims at ranking the "bad" (i.e., likely misclassified) examples higher than the good ones; but this is of course an inessential difference.

As a measure of ranking quality we will choose mean average precision (MAP), which in our context is defined as follows. Let $r_{j}(\widehat{T r})$ be the ranking for class $c_{j}$, realized according to TDC technique $r$, of the perturbed training set $\widehat{T r}$, and let $\left[r_{j}(\widehat{T r})\right]_{k}$ be a binary predicate that returns 1 if the example at the $k$-th position in $r_{j}(\widehat{T r})$ is perturbed for class $c_{j}$, and 0 otherwise. We define the precision at $n$ of $r_{j}(\widehat{T r})$ as

$$
P_{n}\left(r_{j}(\widehat{T r})\right)=\frac{1}{n} \sum_{k=1}^{n}\left[r_{j}(\widehat{T r})\right]_{k}
$$

We then define the average precision of $r_{j}(\widehat{T r})$ as

$$
A P\left(r_{j}(\widehat{T r})\right)=\frac{\sum_{k=1}^{|\widehat{T r}|} P_{k}\left(r_{j}(\widehat{T r})\right) \cdot\left[r_{j}(\widehat{T r})\right]_{k}}{\sum_{k=1}^{|\widehat{T r}|}\left[r_{j}(\widehat{\operatorname{Tr}})\right]_{k}}
$$

The mean average precision (MAP) of technique $r$ on $\widehat{T r}$ is finally defined as

$$
M A P(r(\widehat{T r}))=\frac{1}{|C|} \sum_{c_{j} \in C} A P\left(r_{j}(\widehat{T r})\right)
$$

Aside from a measure of TDC effectiveness we also need a measure of MLTC effectiveness, so as to determine the effectiveness gains in classification obtained if TDC is performed. For this purpose we have used the well-known $F_{1}$ function, in both its microaveraged $\left(F_{1}^{\mu}\right)$ and macroaveraged $\left(F_{1}^{M}\right)$ variants.

Section 4.2 reports the results of our experiments with the three TDC techniques of Section 3, the two different perturbation techniques, different perturbation ratios, and different datasets $\Omega$.

\subsection{The Datasets}

In our experiments we have used the REUTERS-21578 and RCV1-V2 datasets. REUTERS-21578 is probably still the most widely used benchmark in MLTC research. It consists of a set of 12,902 news stories, partitioned (according to the "ModApté" split we have adopted) into a training set of 9,603 documents and a test set of 3,299 documents. The documents are labelled by 118 categories; in our experiments we have restricted our attention to the 115 categories with at least one positive training example. Reuters CoRpus Volume 1 version 2 (RCV1-V2) is a more recent MLTC benchmark made available by Reuters and consisting of 804,414 news stories produced by Reuters from 20 Aug 1996 to 19 Aug 1997. In our experiments we have used the "LYRL2004" split, defined in [3, in which the (chronologically) first 23,149 documents are used for training 
Table 1. Mean average precision (MAP) of the three TDC techniques (CON, NN, $\mathrm{COM}$ ) on the full set of classes (top 4 rows) and on the 30 most infrequent classes (bottom 4 rows) of REUTERs-21578 (left) and RCV1-V2 (right). Boldface indicates the best performer for a given combination of perturbation ratio $(p)$, perturbation method, and dataset.

\begin{tabular}{|c|c|c|c|c|c|c|c|c|c|c|c|c|c|}
\hline & \multirow[b]{3}{*}{$p$} & \multicolumn{6}{|c|}{ REUTERS-21578 } & \multicolumn{6}{|c|}{ RCV1-V2 } \\
\hline & & \multicolumn{3}{|c|}{ Random } & \multicolumn{3}{|c|}{ Targeted } & \multicolumn{3}{|c|}{ Random } & \multicolumn{3}{|c|}{ Targeted } \\
\hline & & $\mathrm{CON}$ & $\mathrm{NN}$ & $\mathrm{COM}$ & $\mathrm{CON}$ & NN & $\mathrm{COM}$ & $\mathrm{CON}$ & NN & $\mathrm{COM}$ & $\mathrm{CON}$ & NN & $\mathrm{COM}$ \\
\hline & .001 & .596 & .458 & .305 & .510 & .369 & .152 & .232 & .238 & .072 & .357 & .082 & .125 \\
\hline & .010 & .653 & .771 & .517 & .608 & .525 & .206 & .752 & .542 & .566 & .519 & 376 & .194 \\
\hline 承 & .050 & .968 & .907 & .808 & .677 & 621 & .301 & .927 & .777 & .801 & .672 & 512 & .417 \\
\hline 勾 & .100 & .973 & .961 & .874 & .665 & .634 & 449 & .945 & .865 & .804 & .658 & .593 & .520 \\
\hline at & .001 & .748 & .790 & .401 & .648 & 681 & .100 & .222 & .225 & .099 & .323 & .101 & .104 \\
\hline$\vec{z}$ & .010 & .674 & .966 & .599 & .581 & .670 & 153 & .702 & .476 & .533 & .435 & 375 & .275 \\
\hline 0 & .050 & .982 & .992 & .812 & .647 & .701 & 268 & .896 & .716 & .747 & .608 & .427 & 479 \\
\hline & .100 & .981 & .985 & .886 & .673 & .651 & 455 & .919 & .845 & .760 & .613 & .523 & .588 \\
\hline
\end{tabular}

and the other 781,265 are used for testing. Of the 103 "Topic" categories, in our experiments we have restricted our attention to the 101 categories with at least one positive training example.

In all the experiments discussed in this paper stop words have been removed, punctuation has been removed, all letters have been converted to lowercase, numbers have been removed, and stemming has been performed by means of Porter's stemmer. Word stems are thus our indexing units; since MP-BoosT requires binary input, only their presence/ absence in the document is recorded, and no weighting is performed.

\subsection{Results and Discussion}

Table 1 reports MAP values obtained by ranking the perturbed training sets by means of the three TDC techniques (CON, NN, COM). Results are reported for the full set of classes and for the 30 most infrequent classes of both REUTERS21578 and RCV1-V2. The reason we pay special attention to the most infrequent classes is that they are usually the classes for which standard supervised learning techniques produce the lowest classification accuracy, which means that they are the classes which are most in need of effectiveness improvement, by TDC or other technique: a user might typically engage in TDC for these highly problematic classes and forget about the classes for which high enough accuracy has already been achieved.

In all the experiments MP-Boost has been run with a number $S$ of iterations fixed to 1,000. For the NN technique, as the $\operatorname{sim}(\cdot, \cdot)$ measure of inter-document similarity we have used the cosine of the angle between the $t$ fidf vectors of the two documents. For the same technique we have used the value $k=45$, since in using $k$-NN as a learning device for TC Yang 4 has found this value to yield the best effectiveness and has found negligible differences between values of $k \in[30,65]$; we defer careful optimization of the $k$ parameter to further work. 
A "trivial" baseline to the results of Table1 is the expected MAP value of the random ranker $(\mathrm{RR})$. Detailed combinatorial analysis shows that this is equal to

$$
M A P(R R(\Omega))=\frac{m-1}{n-1}+\frac{(n-m) H_{n}}{n(n-1)}
$$

where $m$ is the number of relevant (in our case: misclassified) examples in the document set $\Omega, n$ is the total number of examples in $\Omega$, and $H_{n}$ denotes the $n$-th harmonic number (i.e., $H_{n}=\sum_{k=1}^{n} \frac{1}{k}$ ). Actual computation of this formula shows that $M A P(R R(\Omega))$ is approximated by $\frac{m}{n}$ (and in an especially accurate way for large values of $n$ ), which in our case coincides with the perturbation ratio $p=\frac{m}{|T r|}$. Since for all of our datasets and perturbation ratios approximating Equation (5) to the third decimal digit exactly yields $p$, the first column of Table1 1also indicates the trivial baseline for the experiments in the corresponding row.

There are several insights that can be gained from observing the results of Table 1. The first observation is that, since picking training examples at random is the only method one can adopt when wanting to perform TDC, unless equipped with a specific TDC technique such as CON, NN or COM, the improvements that the three TDC techniques display in Table 1 over the baseline of Column 1 is noteworthy.

A second observation is that, with few exceptions and all other things being equal, each technique performs better for random perturbation than for targeted perturbation. This is intuitive, since misclassified training examples inserted at random in the training set tend to be easier to spot; conversely, in targeted perturbation we corrupt the label of borderline examples, which are then much more difficult to identify for any technique.

The third observation is that, among the three competing TDC techniques, while there is no clear winner, there is certainly one clear loser, namely, the COM technique, which in almost all situations obtains results inferior (and often radically so) to $\mathrm{CON}$ and $\mathrm{NN}$. We think that the reason for the bad performance of COM may be found in the fact that MP-BoosT generates a committee of classifiers that are not independent of each other. Indeed, each member $\hat{\Phi}_{s}^{j}$ of the committee strongly depends on the previously generated member $\hat{\Phi}_{s-1}^{j}$, since the former is generated according to the distribution resulting from applying $\hat{\Phi}_{s-1}^{j}$ to $\mathrm{Tr}$. As a consequence, agreement is probably not something one could reasonably expect from the members of this kind of committee, since sharp disagreement may derive from reasons different from a bad label, such as the different emphasis that the different members place, by construction, on a given training example.

Leaving COM aside, we may observe that neither CON nor NN systematically outperform the other. CON tends to be the better technique on the RCV1-V2 dataset, while the situation is less clearcut on REUTERs-21578; similarly, CON tends to outperform NN on the full set of classes of each dataset, while when we analyse the behaviour of the two techniques on the 30 most infrequent classes of each dataset there is no clear winner. All in all, both techniques turn out to be respectable contenders, often achieving (sometimes surprisingly) high MAP values in absolute terms. 
Table 2. Micro- and macro-averaged $F_{1}$ values for the full set of classes (top 5 rows) and for the 30 most infrequent classes (bottom 5 rows) of REUTERs-21578 (left) and RCV1-v2 (right) after random or targeted perturbation

\begin{tabular}{|c|c|c|c|c|c|c|c|c|c|}
\hline & & & REU & 1578 & & & & & \\
\hline & & & & & & & & & \\
\hline & $p$ & $F_{1}^{\mu}$ & $F_{1}^{M}$ & $F_{1}^{\mu}$ & $F_{1}^{M}$ & $F_{1}^{\mu}$ & $F_{1}^{M}$ & $F_{1}^{\mu}$ & $F_{1}^{M}$ \\
\hline$\theta$ & .000 & .852 & .606 & .852 & .606 & .572 & .423 & .572 & .423 \\
\hline 웡 & .001 & .822 & .356 & .821 & .448 & .557 & .368 & .558 & .354 \\
\hline 고 & .010 & .583 & .227 & .632 & .254 & .348 & .224 & .441. & .324 \\
\hline 5 & .050 & .138 & .074 & .209 & .094 & . 105 & .096 & .211 & . 160 \\
\hline & . 100 & .064 & .047 & .116 & .061 & .050 & .064 & . 137 & . 107. \\
\hline & .000 & .373 & .245 & .373 & .245 & .164 & .062 & .164 & .062 \\
\hline 象 & .001 & 190 & . 114 & .139 & .137 & . 102 & .044 & .038 & .035. \\
\hline 4 & .010 & .038 & .036 & .056 & .052 & .025 & .024 & .063. & .039 \\
\hline প & 050 & .004 & .004. & .011 & .011 & .006 & .005 & .015. & .014 \\
\hline & . 100 & .002 & .002. & .006 & .005 & .005 & .003 & .010. & .008 \\
\hline
\end{tabular}

A fourth insight we can gain by looking at Table 1 is that MAP tends to increase with the perturbation ratio $p$, and may reach extremely high values for high values of $p$. This is very good news, since this means that if we have reasons to believe that our training set is extremely low-quality, we know that our time in cleaning it will not be wasted, since these techniques will place almost all the bad examples near the top of the ranking.

Table 2 reports instead the micro- and macro-averaged $F_{1}$ values obtained before and after perturbation; this is an indication of the improvement in classification effectiveness one obtains by performing TDC if the original training set contains noise at the perturbation ratios indicated. Results are reported for the full set of classes and for the 30 most infrequent classes of our two datasets.

One insight that this table allows to gain is that random perturbation is usually more damaging to effectiveness than targeted perturbation, and this fact tends to become evident as the perturbation rate increases. That targeted perturbation may have less disruptive effects is intuitive, since TP introduces mislabellings on documents that are likely borderline examples anyway, i.e., documents that two human annotators might legitimately label in different ways. Mislabelling them may hurt classification accuracy in the thin region of document space close to the surface that separates the positives from the negatives, but does not affect accuracy elsewhere. Conversely, random perturbation may have effects anywhere in document space, and may seriously mislead the classifiers even on cases that would be clearcut otherwise.

A second observation that immediately jumps to the eye is that the decrease in effectiveness deriving from perturbation is noteworthy even for very modest perturbation rates (e.g., .001), and becomes disastrous even for slightly less modest ones (e.g., .010). For instance, for a .001 targeted perturbation rate removing the mislabellings from the REUTERS-21578 training set makes $F_{1}^{\mu}$ jump

- from .821 to .852 for the full set of classes. This is a $3 \%$ relative improvement, that in the '90s has taken years of improvement in TC technology to achieve. 
This shows that one mislabelled document in a thousand can single-handedly defy the efforts of many TC researchers at improving effectiveness;

- from .139 to .373 for the 30 most infrequent classes, a $168 \%$ relative improvement. It is not hard to see why the effect of even a few misclassified training examples on the classification accuracy for infrequent classes can be so large. Given a class with very few positive training examples, mislabelling even one or a handful negatives as positives can severely corrupt the set of positive training examples, while mislabelling even one or a handful of positives as negatives has the double effect of depleting the already slim set of positive examples and confusing the learner by presenting it with negative training documents that are very similar to the remaining positive ones.

These two observations hold to an even higher degree for $F_{1}^{M}$; similar observations also hold for random perturbation and RCV1-V2. For reasons of space we do not separately report the results on the $(|C|-30)$ most frequent classes of our two datasets. In a nutshell, on these classes the decrease in $F_{1}^{\mu}$ is very similar to the decrease on the full set of classes (since $F_{1}^{\mu}$ is mostly influenced by the behaviour on the most frequent classes), while the decrease in $F_{1}^{M}$ is smaller than the decrease in the full set of classes (since $F_{1}^{M}$ is equally influenced by all the classes in $C$ ).

Note that Table 2 only gives us a picture of the improvement that might be obtained by cleaning the entire training set. Aside from probably being infeasible in many real-world situations, this is something that would defy the purpose of the TDC techniques we have presented. A study should thus be performed that, for any combination of TDC technique, perturbation method, perturbation ratio, and dataset, plots the effectiveness of the classifiers generated after TDC has been performed, as a function of $K$, the number of top-ranked training examples that the human annotator has double-checked for misclassifications. This is obviously a daunting experimentation, since for each such combination and each value of $K$ the classifiers should be retrained from scratch and the test examples should be reclassified anew. In Table 3 we provide a sample such

Table 3. Micro- and macro-averaged $F_{1}$ values for the full set of classes (top 5 rows) and for the 30 most infrequent classes (bottom 5 rows) of REUTERs-21578 with classifiers trained after performing TDC by means of the CON technique with $K=100$

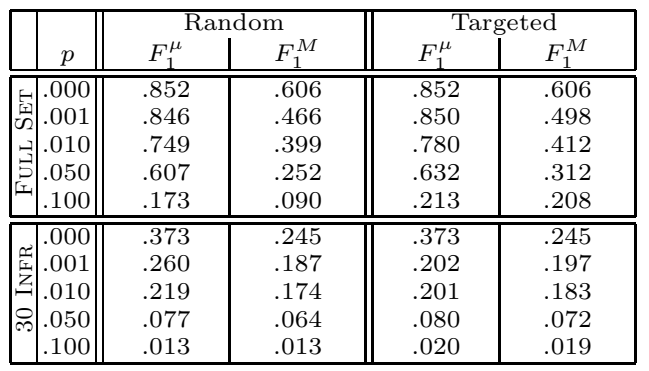


experiment, in which for different perturbation methods and ratios we test the effectiveness values resulting, on REUTERS-21578, from performing TDC by the CON technique and "un-perturbing" the perturbed documents found at the top $K=100$ positions in the ranking. For instance, with targeted perturbation and $p=.001$, the MAP value of .510 that CON obtains guarantees (see Table1) that $F_{1}^{\mu}$, that perturbation had brought down from .852 to .821 (see Table 2), jumps back to .850 , and that $F_{1}^{M}$, that perturbation had brought down from .606 to .448 , jumps back to .498 . All these results are indicative of the fact that TDC is an important and cost-effective way of improving accuracy for all the datasets of less-than-perfect quality of annotation.

\section{Related Work}

Several works have used TDC in learning tasks other than TC, especially within the realm of computational linguistics. Some of these works use task-independent TDC techniques while others do not. Among the former, 56 use the DIS technique discussed at the end of Section 3, while [7] uses a technique analogous to DIS that exploits the characteristics of SVMs. Other works use instead taskspecific techniques; for instance, in a POS-tagging application 8] top-ranks multiple occurrences of the same word that have been classified with different parts of speech in similar linguistic contexts, a technique that is obviously applicable to POS-tagging only and not to tasks such as TC. To the best of our knowledge the only work that deals with TDC in the context of TC is [9. The proposed method consists in training an SVM, removing from the training set the support vectors that the SVM has identified, training a naive Bayesian classifier on the modified training set, and reclassifying the removed support vectors with this classifier, declaring mislabelled the support vectors whose original label does not match the newly assigned label. The intuition behind this technique is that if a training example has a wrong $c_{j}$-assignment, then it likely ends up being a support vector for the generated classifier. Unlike our techniques, this technique is strictly learner-dependent, since it only works with SVMs as learners. Additionally, the method is only limited to cleaning the support vectors; our method examines (and ranks) instead the entire training set; as a result, experimentally comparing the technique of [9] with ours would be problematic.

All of the works above adopt an a posteriori evaluation methodology, i.e., they perform no training set perturbation, and evaluate their techniques by ranking the original training sets and then asking human annotators to look for misclassified examples throughout the first $k$ ranks, thus reporting precision-at- $k$ results. We prefer the a priori evaluation methodology, since (i) it allows us to work with different perturbation ratios, thus addressing the fact that different applications may be characterized by different levels of quality in their data; (ii) it is exempt from evaluator bias, which the a posteriori methodology especially suffers from when (as is frequently the case) it is the authors themselves that engage in post-checking the results; (iii) it allows to compute MAP, while the $a$ posteriori methodology only allows to compute precision for a specific, usually 
low value of $k$ (i.e., the misclassified items from the $(k+1)$-st position onwards have no impact on the evaluation); and (iv) it allows one researcher to replicate the results of the other, while the a posteriori methodology does not.

Finally, let us note that the COM technique is somehow reminiscent of the query-by-committee active-learning method (see e.g., [10]), in which unlabelled examples (and not labelled ones, as in our case) are ranked for human annotation in decreasing order of the disagreement among a committee of classifiers that try to classify them. As a measure of disagreement, [10] uses entropy. We have instead proposed using standard deviation, since entropy can only take into account the binary decisions of the various classifiers, and not the real-valued confidence in their decision; conversely, standard deviation can naturally account for predictions expressed as real numbers, and is thus a better fit in our case.

\section{Conclusions}

We have tested three techniques for TDC on two popular MLTC benchmarks, checking their ability at spotting and top-ranking a set of training examples whose class assignment we have purposefully corrupted for experimental reasons. This experimental protocol allows to conveniently study in vitro the behaviour of these TDC techniques, and to precisely measure the relative merits of the various techniques by means of evaluation measures, such as MAP, standard in the field of ranked retrieval. Studying three TDC techniques with two different perturbation models, at five different perturbation levels, across two datasets (one of which consisting of more than 800,000 documents), and studying both the quality of the resulting rankings and the increase in effectiveness that carrying out TDC may bring about, our work probably qualifies as the first truly-large scale experimentation of TDC in either computational linguistics or IR.

Our experimental results show that two techniques, the confidence-based technique and the nearest neighbours technique, achieve good MAP values across different settings deriving from the choice of different datasets, different class frequency, different perturbation ratios, and different types of perturbation, but also show that neither one clearly outperforms the other. A further result of this paper is that a fourth technique, which had been proposed before and which was specific to boosting-based learners, is equivalent to the confidence-based technique proposed here, which is instead applicable to all learners equipped with a notion of confidence in the classification decision. Our results also show that TDC is important, since they show that even a single misclassified example in a thousand training examples can bring about deteriorations in effectiveness that are simply noteworthy in general, and are no less than dramatic for the most infrequent classes and for macroaveraged $F_{1}$ in general.

Note also that TDC techniques are important not only for training data cleaning, but also for cleaning generic sets of labelled text: the very same techniques discussed here might be applied by a human annotator in order to clean a manually annotated text corpus (e.g., the entire RCV1-V2), regardless of the fact that the entire corpus is then going to be used for training a text classifier. For 
instance, this is useful for cleaning test sets, since incorrectly labelled test examples prevent the accurate measurement of effectiveness, but it is also useful for cleaning labelled datasets produced within organizations that entirely rely on manual classification.

\section{References}

1. Esuli, A., Fagni, T., Sebastiani, F.: MP-Boost: A multiple-pivot boosting algorithm and its application to text categorization. In: Crestani, F., Ferragina, P., Sanderson, M. (eds.) SPIRE 2006. LNCS, vol. 4209, pp. 1-12. Springer, Heidelberg (2006)

2. Schapire, R.E., Singer, Y.: Boostexter: A boosting-based system for text categorization. Machine Learning 39(2/3), 135-168 (2000)

3. Lewis, D.D., Yang, Y., Rose, T.G., Li, F.: RCV1: A new benchmark collection for text categorization research. Journal of Machine Learning Research 5, 361-397 (2004)

4. Yang, Y.: An evaluation of statistical approaches to text categorization. Information Retrieval 1(1/2), 69-90 (1999)

5. Abney, S., Schapire, R.E., Singer, Y.: Boosting applied to tagging and PP attachment. In: Proceedings of the 1999 Joint SIGDAT Conference on Empirical Methods in Natural Language Processing and Very Large Corpora (EMNLP/VLC 1999), College Park, US, pp. 38-45 (1999)

6. Shinnou, H.: Detection of errors in training data by using a decision list and Adaboost. In: Proceedings of the IJCAI 2001 Workshop on Text Learning Beyond Supervision, Seattle, US (2001)

7. Nakagawa, T., Matsumoto, Y.: Detecting errors in corpora using support vector machines. In: Proceedings of the 19th International Conference on Computational Linguistics (COLING 2002), Taipei, TW, pp. 1-7 (2002)

8. Dickinson, M., Meurers, W.D.: Detecting errors in part-of-speech annotation. In: Proceedings of the 10th Conference of the European Chapter of the Association for Computational Linguistics (EACL 2003), Budapest, HU, pp. 107-114 (2003)

9. Fukumoto, F., Suzuki, Y.: Correcting category errors in text classification. In: Proceedings of the 20th International Conference on Computational Linguistics (COLING 2004), Geneva, CH, pp. 868-874 (2004)

10. Argamon-Engelson, S., Dagan, I.: Committee-based sample selection for probabilistic classifiers. Journal of Artificial Intelligence Research 11, 335-360 (1999) 